\title{
e-Carteirinha: O Documento Digital do Estudante
}

\author{
Matheus Dias Vieira ${ }^{1}$, Ewerton R. Andrade ${ }^{1,2}$ \\ ${ }^{1}$ Instituto Federal de Educação, Ciência e Tecnologia de Rondônia (IFRO) \\ ${ }^{2}$ Grupo de Pesquisa em Segurança, Algoritmos, Computação e Inovação (SACI) \\ Departamento Acadêmico de Ciência da Computação (DACC) \\ Fundação Universidade Federal de Rondônia (UNIR) \\ Porto Velho - RO, Brasil \\ matheuspvh13@gmail.com, ewerton.andrade@\{ifro.edu.br|unir.br\}
}

\begin{abstract}
Resumo. Seguindo a tendência de disponibilização de documentos digitais de identificação, o presente trabalho visa criar uma versão digital da Carteira de Identificação Estudantil (CIE) para estudantes do Instituto Federal de Rondônia (IFRO). Dentro das instituições de ensino, este documento é popularmente chamado de "carteirinha", por este motivo, esta nova versão vem sendo chamada de "e-Carteirinha". Com a implantação desta solução, espera-se que: (I) seja diminuído o período de espera para a confecção deste documento de identificação, (II) seja necessário menos servidores/colaboradores para sua emissão, (III) haja um acréscimo de segurança em razão dos mecanismos implementados nesta nova versão digital do documento, (IV) se estabeleça uma estrutura que permita extensão e comunicação com novos sistemas. Para isto, a solução foi desenvolvida nativamente para Android utilizando a linguagem Java e a ferramenta Android Studio, juntamente com bibliotecas de persistência, segurança da informação e comunicação e desenvolvimento web.
\end{abstract}

\section{Introdução}

O uso de dispositivos móveis inteligentes (smartphones, em inglês) vem crescendo anualmente no Brasil e no mundo. Dados levantados pelo Global Mobile Consumer Survey, pesquisa anual realizada pela Deloitte Touche Tohmatsu Limited, empresa especializada em consultoria nas áreas de Marketing e Tecnologia, revelam que 92\% dos brasileiros entrevistados utilizam dispositivos móveis como principal meio de comunicação e acesso a informação [DELOITTE 2018]. Complementarmente, segundo pesquisas de utilização de recursos de Tecnologia da Informação, têm-se que o Brasil possui mais de 420 milhões de dispositivos móveis conectados à Internet, desse total, cerca de 235 milhões são smartphones, o que representa mais de um aparelho habilitado por habitante [Meirelles 2019].

Com este advento, vários dispositivos tecnológicos foram abandonados, e suas funcionalidades foram incorporados aos dispositivos móveis inteligentes. Até mesmo artefatos tradicionalmente analógicos (e.g., calendários, blocos de notas, livros, entre outros) tornaram-se aplicações destes aparelhos. Esse aumento de funcionalidade dos dispositivos móveis, aliado ao crescimento das tecnologias de segurança da informação, propiciou a criação e popularização de versões digitais de vários documentos de identificação.

Mesmo com toda comodidade trazida por essa inovação, no Brasil, somente o Título de Eleitor [TSE 2017], a Carteira Nacional de Habilitação [BRASIL 2017], o Ca- 
dastro de Pessoa Física [BRASIL 2018] e a Carteira de Trabalho e Previdência Social [BRASIL 2015] podem ser acessadas por meio de aplicações móveis.

Dentre os documentos que não possuem uma versão para dispositivos móveis normatizada, destaca-se a Carteira de Identificação Estudantil (CIE), utilizada pela comunidade estudantil de todo Brasil. A "carteirinha de estudante", como é popularmente conhecida, identifica os alunos matriculados - podendo garantir acesso a áreas restritas da instituição a qual está vinculado -, além de assegurar descontos em comércios conveniados a sua instituição de ensino bem como em estabelecimentos com "meia-entrada" garantida por lei [BRASIL 2013].

Recentemente o Governo Federal buscou regulamentar a CIE nacional em formato digital [BRASIL 2019], esta iniciativa ficou conhecida como "ID Estudantil" [BRASIL 2020]. Apesar de sua grande popularidade inicial, pois segundo informações oficiais 424.787 documentos digitais foram emitidos [BRASIL 2020], este formato de documento foi descontinuado em virtude da Medida Provisória que o criou ter perdido a validade por não ter sido analisada pelo Congresso Nacional no prazo regimental de 120 dias [BRASIL 2020].

Os documentos emitidos não perderam a validade [BRASIL 2020], porém, o Governo Federal não poderá emitir mais documentos através dessa plataforma. Contudo, toda instituição de ensino possui autonomia para emitir ou credenciar estabelecimentos para tal [BRASIL 2013], desde que obedeçam os padrões estabelecidos no ordenamento jurídico [BRASIL 2013, IFRO 2016].

\section{Motivação e Justificativa}

Valendo-se desta prerrogativa de autonomia, no Instituto Federal de Rondônia cada campus é responsável por realizar a emissão da CIE para seus discentes no prazo de 60 dias a partir do início do ano letivo [IFRO 2016]. No entanto, o alto número de estudantes, em cada uma de suas unidades, nas mais diferentes modalidades e níveis, torna o prazo impraticável. Sendo inevitável que a entrega da carteira de identificação acabe sendo realizada meses após o prazo estipulado, causando inúmeros transtornos ao corpo discente.

Tentando contornar o problema do prazo, anualmente, servidores/colaboradores são designados para uma comissão responsável pela emissão da CIE. Apesar de aumentar a velocidade de produção do documento, a convocação destes servidores causa problemas em seus setores de origem, pois a redução do quadro sobrecarrega os demais servidores/colaboradores e diminui a eficiência dos trabalhos técnico-administrativos. Ou seja, tentando solucionar um problema ocasiona-se outro.

Além disso, vale destacar que apenas o campus Porto Velho Calama gastou, no ano de 2018, R \$1.794,00 (mil setecentos e noventa e quatro reais) somente com a aquisição de cartões de PVC para a emissão da Carteira de Identificação estudantil (CIE) [IFRO 2018], este é, em média, o valor empenhado anualmente para esse fim. A quantia citada é majorada pelos custos de impressão do documento (e.g., suprimentos para impressora, manutenção do equipamento, dentre outros). Outro fator que pode aumentar o valor gasto na emissão são as possíveis falhas e erros no momento da impressão, que podem inutilizar matéria-prima e demandar mais tempo para a reimpressão e alocação de recursos extras para a reposição do material perdido. 
Em contrapartida, segundo levantamento do governo federal [BRASIL 2020], estima-se que o custo para emissão da CIE digital ficaria em torno de $\mathrm{R} \$ 0,15$. Podendo chegar a valores ainda menores em caso de uma maior emissão de documentos. O que representaria uma economia significativa para os cofres públicos.

Desta forma, com a economia esperada com a adoção da e-Carteirinha, esperase que sejam alocados recursos para a realização de melhorias na infraestrutura de salas de aula e laboratórios ou aumentar a oferta de bolsas e auxílios à pesquisa científica e tecnológica, dentre outras ações de investimento em ensino, pesquisa e extensão.

Adicionalmente, frisa-se que a carteira de identificação física carece de mecanismos de segurança que comprovem a autenticidade e integridade das informações nela contidas [STF 2014]. Essa deficiência facilita a falsificação, o que pode contribuir para o descrédito do documento junto aos parceiros da instituição de ensino, uma vez que qualquer pessoa com material gráfico adequado pode emitir uma réplica desta carteira de identificação. Essas carências tornam o documento pouco confiável e, consequentemente, desvirtuam o propósito e o investimento empregado em sua confecção.

\section{Objetivo}

Para contornar os problemas mencionados anteriormente, levantou-se a proposição de desenvolver um aplicativo para dispositivos móveis que permita ao discente do Instituto Federal de Rondônia acessar uma versão digital da "carteirinha de estudante" com toda a praticidade do documento impresso, acrescido da comodidade e serviços básicos de segurança fornecidos por este tipo de plataforma.

Uma das bases do desenvolvimento da "e-Carteirinha", a CIE digital, foi buscar por tecnologias que garantissem a autenticidade, integridade e irretratabilidade (também chamada de não repúdio) das informações presentes no documento.

Além disso, para não contrastar com os dispositivos legais já estabelecidos, a "eCarteirinha" deverá ser emitida conforme modelo padrão estabelecido nacionalmente e regulamentado pela Reitoria do Instituto Federal de Rondônia [IFRO 2016, BRASIL 2013].

Seguindo estes preceitos, espera-se que o novo documento seja integrado às funcionalidades do "IFRO Mobile" [IFRO 2019], aplicativo oficial do Instituto. Conjectura-se, ainda, que a adesão a "e-Carteirinha" seja simples, uma vez que os discentes já estão familiarizados com a aplicação institucionalizada pelo IFRO.

\section{Descrição da solução e dos métodos}

\subsection{Método científico}

O método adotado durante o desenvolvimento desta solução foi o da pesquisa exploratória [Wazlawick 2008], uma vez que os autores não tinham necessariamente uma noção de todas especificidades que encontrariam. Desta forma, além de buscas de referências em bases científicas e tecnológicas, também foram realizadas entrevistas e reuniões com potenciais usuários da "e-Carteirinha" (i.e, estudantes, técnicos administrativos e gestores).

Assim, conforme os autores foram tomando conhecimento do processo de emissão da Carteira de Identificação Estudantil (CIE) do IFRO, bem como das técnicas atualmente utilizadas, os autores foram, então, desenvolvendo estratégias de pesquisa e desenvolvendo a solução conforme as particularidades se apresentavam. 


\subsection{Mapeamento do fluxo de emissão}

Para entender o processo de emissão adotado atualmente, além da leitura e compreensão dos procedimentos estabelecidos no Regulamento para emissão das carteiras de identificação estudantil do IFRO [IFRO 2016], realizaram-se entrevistas com servidores do Departamento de Assistência ao Educando (DEPAE) e da Coordenação de Registros Acadêmicos (CRA), ambos do campus Porto Velho Calama.

Desta forma, sanou-se as dúvidas e levantou-se todas informações sobre o processo de coleta de dados dos estudantes, bem como esclareceu-se o fluxo de atividades necessárias para a emissão da carteira estudantil impressa.

\subsection{Escolhas de projeto}

A "e-Carteirinha" foi desenvolvida para dispositivos com sistema operacional Android $4.0 \mathrm{ou}$ superior. A escolha desta plataforma deu-se em virtude do aplicativo institucional "IFRO Mobile" utilizar estas mesmas especificações e, conforme já mencionado, pretende-se integrar a aplicação desenvolvida ao aplicativo institucional.

Após a finalização do projeto e publicação deste trabalho, tal integração será realizada pela equipe da Coordenação de Desenvolvimento de Sistemas (CDSIS) do IFRO. Contudo, seu código fonte pode ser acessado, auditado e adaptado as necessidades de qualquer instituição de ensino através do repositório: https://bitbucket.org/ Koalapvh13/novaecarteirinha/.

Além destes fatores, destaca-se que foi realizada uma pesquisa junto ao setor de Tecnologia de Informação do campus Porto Velho Calama e foi constatado que o sistema operacional móvel mais utilizado pela comunidade acadêmica é o Android. Mais especificamente, verificou-se que mais de $75 \%$ dos dispositivos conectados a rede institucional utiliza o sistema operacional Android.

\subsection{Ambiente de desenvolvimento}

O aplicativo foi desenvolvido para rodar nativamente no Android, utilizando a linguagem Java, a ferramenta Android Studio 3.1.4 e conta com técnicas de persistência de dados off-line, utilizando a biblioteca de Mapeamento Objeto-Relacional Room.

Além da biblioteca Room, foram utilizadas as bibliotecas Volley 1.1.1, para realização de requisições http, a ZXING, responsável pela geração do QRCode, e a Altex Image Download, para realizar o download e armazenamento de imagens do servidor.

Vale ressaltar que todas as bibliotecas utilizadas no contexto deste projeto são livres (open-source) e foram utilizadas para otimizar o desenvolvimento do projeto.

\subsection{Coleta de dados dos estudantes}

Os dados utilizados na "e-Carteirinha" são fornecidos por uma API (Application Programming Interface) do próprio Instituto Federal. Nesta API, os dados são buscados no servidor de autenticação Active Directory (AD) e retornados por meio de arquivo JSON (JavaScript Object Notation) para a aplicação.

Para alimentar a base de dados, é realizado o seguinte processo: ao realizar a matrícula, o discente informa vários dados à Coordenação de Registros Acadêmicos (e.g., 
nome, $\mathrm{CPF}$, matrícula, foto, etc.) que, posteriormente, digitaliza os documentos e insere os dados no Active Directory Data Store (serviço de armazenamento do AD). Com isto, fica garantida a autenticidade (uma vez que é realizada por colaboradores da instituição), disponibilidade e a não duplicidade das informações fornecidas pelos alunos (já que estes são alguns do serviços de segurança básicos fornecidos pelo $\mathrm{AD}$ ).

Para acessar as informações gravadas no AD é utilizada a API oficial do IFRO, criada pelos técnicos e analistas da Diretoria de Gestão de Tecnologia da Informação (DGTI), que requer o usuário, no caso o número do CPF, e a senha utilizada pelo estudante no acesso aos sistemas institucionais. Caso ocorra a autenticação dos dados (usuário/senha válido), o sistema retorna um arquivo JSON contendo todas as informações cadastradas do referido aluno. Caso contrário (usuário/senha inválido ou outro tipo de erro), é retornado um arquivo JSON contendo informações sobre o erro.

Caso o AD esteja indisponível (off-line) ou o smartphone esteja sem acesso a Internet, os dados armazenados com o auxílio da biblioteca de Mapeamento ObjetoRelacional Room serão utilizados. Nesta situação, caso o estudante já tenha realizado alguma autenticação válida, os dados armazenados serão utilizados para exibir o documento digital. Caso isto ainda não tenha ocorrido, o documento digital não será gerado e a aplicação exibirá uma mensagem de erro, ficando o serviço indisponível até que o acesso ao servidor de autenticação se reestabeleça.

\subsection{Serviços de segurança da informação}

Visando garantir a autenticidade, a integridade e a irretratabilidade dos documentos emitidos, a aplicação conta com assinatura digital de todos dados do estudante. Para isso, são geradas chaves públicas e privadas utilizando o algoritmo de assinatura digital DSA (Digital Signature Algorithm).

Este algoritmo foi desenvolvido por David Kravitz quando este trabalhava para a NSA (National Security Agency, Agência de Segurança Nacional dos Estados Unidos, em português) [NIST 2013] baseando-se no trabalho dos criptógrafos El Gamal e Claus Schnoor. Ressalta-se que o DSA é um dos algoritmos de assinatura digital mais difundidos da atualidade, além disso, ele é utilizado como padrão por diversas agências governamentais ao redor do mundo [NIST 2013].

Usando a chave privada, os dados do documento e do aparelho (e.g., nome, CPF, matrícula, foto, modelo do aparelho, número do série, etc.) são digitalmente assinados e essa assinatura é colocada em um QRCode, juntamente com sua chave pública. O QRCode gerado é exibido como parte da "e-Carteirinha" e, quando lido, encaminha o usuário para uma página web pública que faz a validação do documento por meio de todos os dados passados como parâmetros (todos dados assinados digitalmente e utilizados na confecção do documento digital). Podendo esta página também ser utilizada como verificador de autenticidade em outros sistemas que necessitem deste tipo de serviço e possuam acesso à Internet.

Assim, toda vez que é acessado, este verificador hospedado em uma página web exibe os dados contidos no QRCode para uma possível inspeção realizada por humanos, e ainda, se comunica com o servido de autenticação oficial (i.g., AD) e realiza uma validação digital e informa caso seja constatada alguma falha de autenticidade ou integridade dos dados fornecidos. Evitando, desta forma, possíveis ataques como 
manipulação/adulteração dos dados assinados digitalmente, cópia e reprodução dos documentos gerados ("prints" da tela), criação de documentos com dados forjados, e qualquer outro tipo de golpe envolvendo dados fraudulentos.

\section{Resultados e discussões}

Apesar da "e-Carteirinha" não estar implantada como um serviço oficial do IFRO em razão dos procedimentos administrativos necessários em qualquer instituição pública (aprovação em conselhos, hospedagem em servidores oficiais, e outras etapas inerentes a qualquer novo serviço institucional de Tecnologia da Informação), já é possível apontar diversos resultados advindos das ações relacionadas ao seu desenvolvimento.

O mapeamento e a normalização de todos os passos do fluxo para emissão da CIE, desde a coleta dos dados até a sua entrega, pode ser considerado consequência direta da análise e levantamento de requisitos do projeto. Este fluxo (discutido na seção 4) pode ser adequado as necessidades de outras instituições, todavia, é essencial que ele seja bem estabelecido, principalmente quanto a coletada de dados dos estudantes, que no caso do IFRO é realizada pela Coordenação de Registros Acadêmicos (seção 4.5).

Outro resultado relacionado à implementação deste trabalho é o estabelecimento de uma API única para acesso aos dados. Dados estes, essenciais para a confecção da Carteira de Identificação Estudantil Digital. Esta medida foi realizada em parceria com a Diretoria de Gestão de Tecnologia da Informação (DGTI) do IFRO para viabilizar o desenvolvimento da "e-Carteirinha" de forma compatível e integrável ao aplicativo oficial, contudo, esta API poderá ser utilizada por futuros projetos e aplicações que necessitarem de acesso a este tipo de informação ou buscarem funcionar de forma integrada.

Destaca-se, ainda, que o desenvolvimento de um protótipo totalmente funcional seja considerado como resultado principal da execução deste projeto, principalmente pela sua capacidade de integração ao aplicativo institucional IFRO Mobile (a Figura 1 ilustra o funcionamento deste protótipo). Isso porque ele não somente viabilizou a criação de uma versão digital da CIE - cômoda e integrável aos demais sistemas do instituto (objetivos principais deste trabalho, seção 3) -, mas também possibilitou a observação de que, utilizando este documento digital, é possível diminuir o número de servidores/colaboradores e o tempo envolvidos no processo de confecção da carteirinha, além de incrementar a autenticidade, integridade e irretratabilidade das informações presentes no documento.

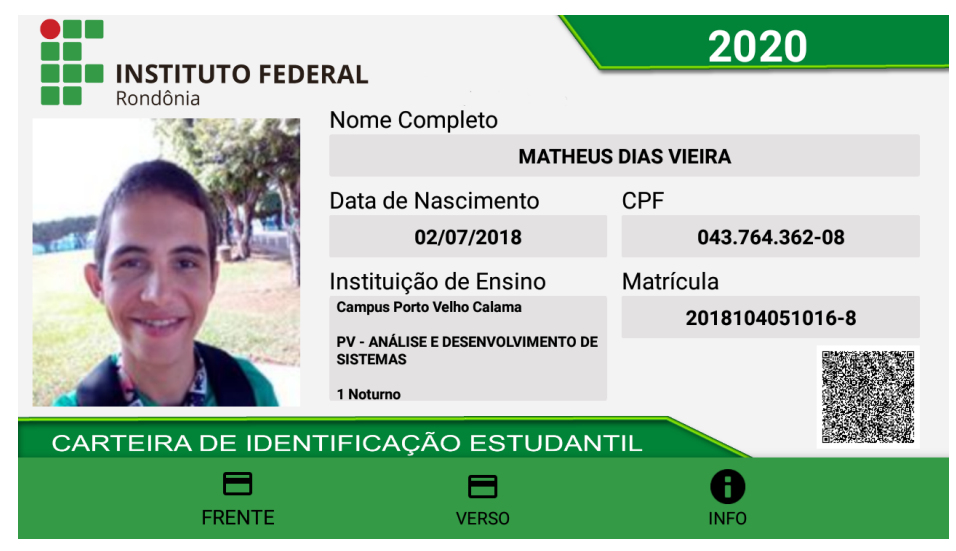

Figura 1. Aplicação "e-Carteirinha" em funcionamento. 
Um outro especto importante do protótipo é o verificador hospedado em uma página web pública (maiores detalhes disponíveis na seção 4.6). Pois, além de realizar a verificação digital da autenticidade e integridade dos dados, também auxilia humanos na identificação manual dos estudantes, e fornece um mecanismo extensível para outros sistemas que necessitem de algum tipo de autenticação dos estudantes. Além disso, salienta-se que, por se tratar de um serviço hospedado em uma página web pública, o verificador funcionará em qualquer dispositivo com acesso a Internet e leitor de QRCode, independentemente do sistema operacional instalado.

Ademais, durante levantamento bibliográfico para extensões do trabalho e aplicações de mecanismos de autenticação modernos (como a carteirinha digital) e, também, no decorrer dos testes realizados pelos autores e alguns estudantes do curso de Análise e Desenvolvimento de Sistemas, verificou-se que a "e-Carteirinha" poderá ser utilizada em outros contextos além da identificação dos estudantes. Por exemplo, somente no ambiente estudantil ela poderá ser utilizada para (mas não limitado): registro de assiduidade e pontualidade dos alunos; acesso à bibliotecas, salas de estudo e laboratórios com travas eletrônicas adaptadas para a tecnologia; empréstimo de livros na biblioteca; acesso e pagamento do restaurante universitário; pagamento de serviços gráficos e de impressão; cartão de débito aceito no comércio interno da instituição; armazenamento de informações acadêmicas; impressões de documentos oficiais em máquinas de atendimento; acesso ao ônibus interno universitário; depósito de auxílios como bolsas de monitoria e iniciação científica e tecnológica; registro de presença em palestras e atividades extracurriculares; e homologação de horas de atividade complementar especial [Chagas 2017].

Por fim, vale ressaltar que Carteiras de Identificação Estudantil físicas continuarão sendo produzidas para atender estudantes que não possuam dispositivos móveis inteligentes ou utilizem aparelhos com especificações não suportadas pelo aplicativo da "eCarteirinha". Todavia, conforme discutido na seção 4.3, estima-se que isto ocorra na minoria dos casos, podendo ocorrer cada vez menos conforme o projeto se popularize e seja refatorado para outras plataformas (e.g., iOS).

\section{Considerações finais}

A principal contribuição deste trabalho foi o desenvolvimento de uma aplicação para dispositivos móveis inteligentes capaz de gerar uma Carteira de Identificação Estudantil Digital. Como resultados relacionados, além do desenvolvimento desta aplicação integrável aos demais sistemas institucionais, também foi possível: mapear e normalizar processos relacionados a emissão da CIE; estabelecer uma API única para acesso aos dados dos estudantes; observar que é possível diminuir o número de servidores/colaboradores e o tempo envolvidos no processo de confecção da carteirinha; além de incrementar a autenticidade, integridade e irretratabilidade das informações presentes no documento.

Adicionalmente, acredita-se que estes resultados contribuam com as medidas de isolamento social necessárias ao combate a vírus desconhecidos, como as que estão sendo adotadas no enfrentamento à pandemia do novo coronavírus (COVID-19).

Como continuidade deste trabalho, espera-se que o Instituto Federal de Rondônia promova a integração do "e-Carteirinha" com o aplicativo institucional IFRO Mobile para que seja possível averiguar outros aspectos de seu funcionamento. Mais especificamente, almeja-se avaliá-lo nas dimensões de (mas não limitado): usabilidade ( $a$ interface e a 
experiência de usuário atendem aos requisitos dos usuário?); acessibilidade (a interface acessível? há restrições para os que possuem necessidades especiais?); e aceitabilidade (os estudantes e a administração sinalizaram positivamente quanto ao uso do aplicativo? que serviços, ou "ecossistema”, podem ser desenvolvidos utilizando o e-Carteirinha?).

\section{Agradecimentos}

Este trabalho recebeu o auxílio financeiro e bolsas de Iniciação Tecnológica (PIBIT) do Conselho Nacional de Desenvolvimento Científico e Tecnológico (CNPq), processo número 23243.008122/2018-24.

\section{Referências}

BRASIL (2013). Lei n ${ }^{\circ} 12.933$, de 26/12/2013. Presidência da República.

BRASIL (2015). Portaria n ${ }^{\circ} 3$, de 26/01/2015. Imprensa Nacional.

BRASIL (2017). Resolução nº 684, de 25/07/2017. Imprensa Nacional.

BRASIL (2018). Certificação digital. Instituto Nacional de Tecnologia da Informação.

BRASIL (2019). Medida provisória 895, de 6/9/2019. Presidência da República.

BRASIL (2020). ID Estudantil. Ministério da Educação. http://idestudantil .mec.gov.br/.

Chagas, J. M. A. N. (2017). Aplicação de tecnologia smart card e RFID no ambiente universitário. Monografia (Bacharel em Engenharia de Produção), Escola Politécnica (EP), Universidade Federal do Rio de Janeiro (UFRJ), Brasil.

DELOITTE (2018). Global Mobile Consumer Survey 2018. Technical report, Deloitte Touche Tohmatsu Limited. https://www2.deloitte.com/br/pt/pages/ technology-media-and-telecommunications/articles/mobile-survey.html.

IFRO (2016). Resolução n ${ }^{0}$ 72/CONSUP/IFRO/2016, de 28 de dezembro de 2016. Conselho Superior do Instituto Federal de Educação, Ciência e Tecnologia de Rondônia. https://portal.ifro.edu.br/images/Campi/Colorado_do_Oeste/Documentos/Resolu\%C3\%A7\%C3\% A30_n\%C2\%BA_72_-_Regulamento_de_Carteira_Estudantil_-_SEI_-_Retificada.pdf.

IFRO (2018). Pregão eletrônico $n^{0}$ 04/2018 - aquisição pincel para quadro branco, refil, tinta e cartão pvc. Instituto Federal de Educação, Ciência e Tecnologia de Rondônia. http://portal.ifro.edu.br/ licitacoes/251-pro-reitorias/proap/licitacoes/campus-guajara-mirim-uasg-158635/ 4585-pe-aquisicao-pincel-para-quadro-branco-refi-tinta-e-cartao-pvc.

IFRO (2019). Ifro mobile. Google Play. https://play.google.com/store/apps/details?id= br.edu.ifro.mobile.app.

Meirelles, F. S. (2019). Pesquisa Anual do Uso de TI. Technical report, Fundação Getulio Vargas - FGV. https://eaesp.fgv.br/ensinoeconhecimento/centros/cia/pesquisa.

NIST (2013). FIPS PUB 186-4: Digital Signature Standard (DSS). Technical report, National Institute of Standards and Technology, NIST, U.S., Washington DC.

STF (2014). Ação Direta de Inconstitucionalidade - 5108. Supremo Tribunal Federal.

TSE (2017). Resolução no 23.537, de 5/12/2017. Tribunal Superior Eleitoral.

Wazlawick, R. S. (2008). Metodologia de pesquisa para ciência da computação. Elsevier, Rio de Janeiro. 